\title{
Location Privacy based on Trusted Computing and Secure Logging
}

\author{
Urs Hengartner \\ Cheriton School of Computer Science \\ University of Waterloo \\ Waterloo ON, N2L 3G1, Canada \\ uhengart@cs.uwaterloo.ca
}

\begin{abstract}
Many operators of cellphone networks now offer locationbased services to their customers, whereby an operator often outsources service provisioning to a third-party provider. Since a person's location could reveal sensitive information about the person, the operator must ensure that the service provider processes location information about the operator's customers in a privacy-preserving way. So far, this assurance has been based on a legal contract between the operator and the provider. However, there has been no technical mechanism that lets the operator verify whether the provider adheres to the privacy policy outlined in the contract. We propose an architecture for location-based services based on Trusted Computing and Secure Logging that provides such a technical mechanism. Trusted Computing lets an operator query the configuration of a location-based service. The operator will hand over location information to the service only if the service is configured such that the service provider cannot get access to location information using software-based attacks. This includes passive attacks, where the provider monitors information flowing into and out of its service, and active attacks, where the provider modifies or injects customer queries to the service. We introduce several requirements that must be satisfied by a location-based service to defend against passive attacks. Furthermore, we present Secure Logging, an auditing mechanism to defend against active attacks.
\end{abstract}

\section{Categories and Subject Descriptors}

C.2.0 [General]: Security and protection

\section{General Terms}

Design, Security

\section{Keywords}

Location-based Services, Timing Attacks, Trusted Platform Module

Permission to make digital or hard copies of all or part of this work for personal or classroom use is granted without fee provided that copies are not made or distributed for profit or commercial advantage and that copies bear this notice and the full citation on the first page. To copy otherwise, to republish, to post on servers or to redistribute to lists, requires prior specific permission and/or a fee.

Secure Comm 2008. September 22-25, 2008, Istanbul, Turkey.

Copyright 2008 ACM 978-1-60558-241-2 ...\$5.00.

\section{INTRODUCTION}

The ubiquity of cellphones has led many operators of cellphone networks to offer location-based services to their customers, allowing these customers to map their current location, learn about interesting, nearby places, locate other cellphone users, etc. Often, an operator outsources the provisioning of a location-based service to a third-party provider. This outsourcing raises privacy concerns. Namely, the knowledge of a person's current or past locations could reveal sensitive information about the person's interests, her health, or her political preferences. Therefore, location information itself is sensitive, and the operator should have assurance that the service provider deals with location information about the operator's customers in a privacy-preserving way, based on a given privacy policy. So far, this assurance has relied on a legal contract between the operator and the provider. However, there has been no technical mechanism that lets an operator verify whether the provider adheres to the privacy policy.

In this paper, we present an architecture for locationbased services that provides such a mechanism. Our architecture exploits Trusted Computing technologies 22 to let an operator gather information about the configuration of the platform that provides a location-based service. The operator hands over location information to the platform only if the platform is configured to implement an outlined privacy policy. Here, we are interested in the most constrained case where the policy states that the service platform must provide its service such that the service provider cannot learn any location information.

Unfortunately, giving a comprehensive guarantee that covers all possible attempts by a service provider to get access to location information is likely impossible. However, we can provide a weaker, but still useful guarantee. Namely, we can guarantee that, using software-based attempts only, the service provider will not be able to learn fine-grained location information. This guarantee is superior to the non-technical guarantees offered by current location-based services.

The usage of Trusted Computing technologies to inspect the configuration of a service has already been suggested 14 . 20. We address two additional challenges. First, the earlier work has not considered how interactions of the service (e.g., a web server) with the backend infrastructure (e.g., a database) could lead to information leaks. We show that, in a passive attack, the service provider could learn location information by observing the composition or the timing of information flowing into and out of the service platform. Second, the earlier work includes an entire application, such 
as a web server consisting of thousands of lines of code, in the computing base that is remotely inspected and subsequently trusted. The size of such an application makes it likely that there is a vulnerability that a service provider could exploit. Therefore, we do not want to include an entire location-based service in the trusted computing base. Instead, only the part that needs direct access to location information (about 400 lines of code for our sample locationbased services) should be included, but not, for example, the access-control part. However, this approach enables active attacks by a service provider, where the provider modifies or injects queries to the service to learn location information.

We make the following contributions:

- We build an architecture for the privacy-preserving processing of location information based on Trusted Computing technologies.

- We give a set of requirements that need to be satisfied to defend against passive attacks by a service provider.

- We present Secure Logging, an auditing mechanism that allows the retroactive detection of active attacks by a service provider.

In the extended version of this paper 11, we present an implementation, security analysis, and performance evaluation of our architecture. We presented a preliminary version of our architecture in a workshop paper 10, omitting the requirements needed to defend against passive attacks, the protocol to validate satisfaction of these requirements, and Secure Logging.

In the rest of this paper, we first present our system and threat models (Section 2). We then introduce our architecture (Section 3). Next, we examine two of its components in more detail, the Trusted Module (Section 4) and Secure Logging (Section 5). We then present a sample location-based service built using this architecture (Section 6). Finally, we survey related work (Section 7).

\section{SYSTEM AND THREAT MODELS}

In our system model, the operator of a cellphone network and the provider of a location-based service are separate entities. There are two infrastructures, the cellphone infrastructure, run by the operator of the cellphone network, and the service infrastructure, run by the provider of the location-based service. The cellphone infrastructure keeps track of the location of cellphones by observing which cell towers a cellphone is connecting to or by directly getting location information from GPS-enhanced cellphones. When asked for by the service infrastructure, typically as a consequence of a query by a cellphone user, the cellphone infrastructure hands over location information, maybe in processed form, to the service infrastructure. Several network operators in the UK, such as Vodafone or Orange, provide their customers' location to service providers, such as mapAmobile 4] or World-Tracker.Com 24. Sprint and Bell Canada use WaveMarket's Family Finder 23 to provide location-based services. Lots of existing location privacy research is also based on this system model $1,7,13,16,18$, 19 .

Our threat model consists of a service provider learning a user's fine-grained location. The provider can perform software-based attacks to extract this information from the service infrastructure, but no hardware-based attacks or attacks based on physical user observation. These attacks are more expensive to perform. Also, defending against software-based attacks still gives us better privacy than what existing location-based services provide. We also assume that a service provider can observe the input and the output flowing into and out of the service platform and modify or inject queries sent to the platform. Finally, for efficiency reasons, we allow the service provider to learn a user's coarse-grained location. As it turns out, this happens only for some location-based services, not all of them.

Our threat model allows a service provider to learn the identity of its users. For some services, such as a service to locate interesting, nearby places, it is straightforward (ignoring billing challenges) to extend our approach such that the service provider does not learn identity information; the cellphone infrastructure simply anonymizes a query before sending it to the service infrastructure. For other services, such as a service to locate nearby friends, where the service infrastructure needs information about friend relationships, the solution is less obvious and topic of future research.

\section{ARCHITECTURE OVERVIEW}

Figure 1 shows our architecture for location-based services. In the cellphone infrastructure, we leave away components not relevant in this paper, such as cell towers. The cellphone infrastructure consists of a set of modules and communication links between these modules, where both the modules and the links are under the control of the network operator. The same applies to the service infrastructure, which is under the control of the service provider. Communication between modules belonging to different infrastructures takes place over TLS with client and server authentication, which avoids sniffing, modification and injection attacks by outsiders.

\subsection{Attestation Queries}

Initially, the Attestation Module in the cellphone infrastructure queries the service infrastructure for the configuration of the Trusted Module (see Section 4.2 for details). It does so by sending an attestation query to the Query Processor Module in the service infrastructure, whose task is to receive different kinds of queries, to ensure that they are authorized, to process them if necessary, and to forward them to another module. In the attestation case, the module forwards the query to the Trusted Module and returns the response ("quote") to the Attestation Module. If this module is satisfied with the reported configuration (i.e., the configuration does not leak location information, see Section 4.1), it will give a symmetric key to the Trusted Module such that the Query Processor Module cannot learn the key. Later, the cellphone infrastructure will use the key for encrypting location information before handing the information over to the service infrastructure. This way, the Trusted Module, but not the Query Processor Module (or the service provider), can decrypt and learn location information.

\subsection{Service and Location Queries}

A cellphone user who wants to access a location-based service sends a service query to the cellphone infrastructure, namely, to the Forwarder Module. The Forwarder Module checks validity of the query based on information in the User Database (e.g., did the user sign up to the location-based 


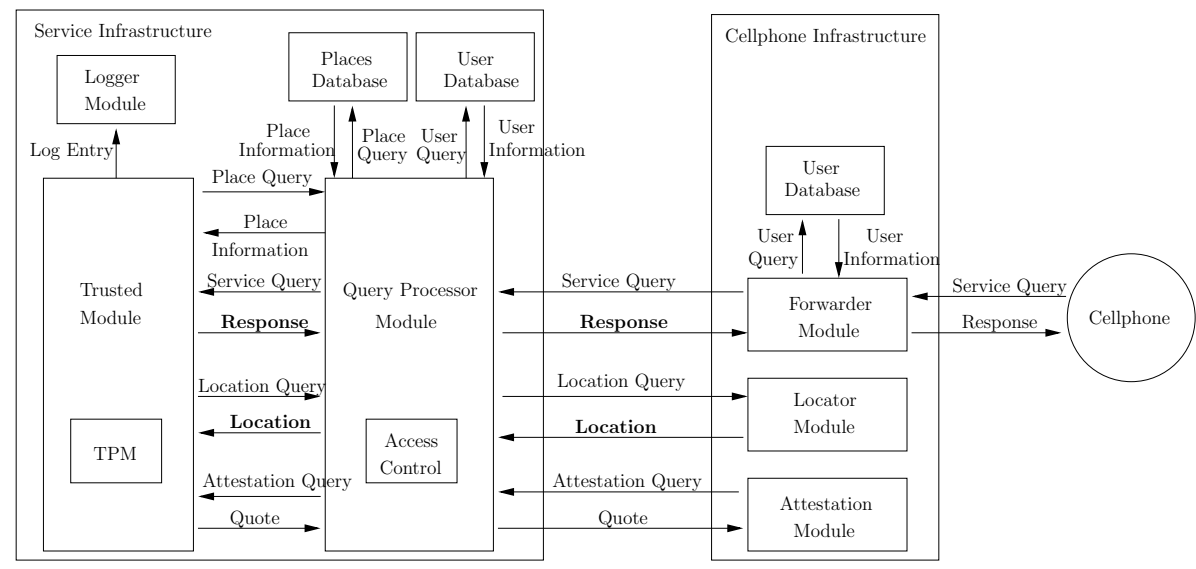

Figure 1: Architecture for location-based services. A cellphone sends a service query via the cellphone infrastructure to the service infrastructure, which uses the Trusted Module for processing the query and generating a response. Items in bold are encrypted (Response and Location).

service?). For accountability reasons, the module then signs the query and sends it to the location-based service, in particular, to the Query Processor Module. This module validates the signature of the query. For some services, such as a service that checks whether a friend is nearby, the module must also ensure that the cellphone user issuing the query is authorized to learn whether the queried person is nearby. Authorization information is stored in the User Database. If the query is authorized, the Query Processor Module sends a location query to the Locator Module in the cellphone infrastructure to retrieve the location information required for processing the query, such as the location of the user's and her friend's cellphone. The Locator Module returns location information only in encrypted form, namely, encrypted with the symmetric key introduced in Section 3.1 Next, the Query Processor Module hands over the location information, the operator's public key, and logging information (see Section 5 to the Trusted Module.

The Trusted Module decrypts the location information. If necessary, as in the case of a query for interesting, nearby places or for traffic conditions, the module asks the Places Database via the Query Processor Module for location-specific information, such as road maps or conditions, weather information, shops, or restaurants. The Trusted Module then generates and signs its response and encrypts it with the operator's public key. To detect misbehaviour by the service provider, the module generates logging information, which it hands over to the Logger Module (see Section 57. The Trusted Module gives its response to the Query Processor Module, which forwards it to the Forwarder Module for decryption and signature checking. Finally, the Forwarder Module forwards the plaintext response to the cellphone.

\section{TRUSTED MODULE}

The Trusted Module is at the core of a location-based service and generates responses to service queries. The module is deployed by the service provider and has access to location information. Nonetheless, the service provider should not have access to this information. As stated in Section 2, we assume that the provider can perform only software-based attacks to gain access to the information. We discuss the set of requirements that need to be satisfied in order to defend against these attacks in Section 4.1. To ensure that a platform actually fulfills these requirements, a network operator uses the concept of remote attestation, which we discuss in Section 4.2

\subsection{Avoiding Information Leaks}

To infer a cellphone's location, the service provider can mount various software-based attacks: First, the provider can observe the input given to the Trusted Module. Second, the provider can observe the output generated by the module. Third, the provider can observe the delay between input and output events. Fourth, the provider can examine the memory used by the module. We now examine each of these attacks.

\subsubsection{Input Attacks}

There are two kinds of input that are given to the Trusted Module and that could reveal a cellphone's location. First, there is actual location information. As discussed in Section 3.1. we require that location information is encrypted before it is given to the Trusted Module. The service provider does not know the decryption key, so the ciphertext is useless to the provider. Second, there is information about places (e.g., their locations), as retrieved from the Places Database. By examining which records the Trusted Module requests from the database, the provider could learn a user's location. We could avoid this attack by including the entire Places Database in the Trusted Module. However, this would drastically increase the size of the trusted computing base, whereas our goal is to keep it as small as possible. As discussed in Section 7. Private Information Retrieval (PIR) schemes also avoid this attack, but tend to be inefficient. We use a compromise that is more efficient, but that lets the service provider learn some information about a user's location. In particular, we require the Trusted Module to cloak a user's location before accessing the Places Database. For example, when the user is at the train station, the module determines a larger area that includes the train station, retrieves information about all the places in the cloaked area from the Places Database, and filters information about places that 
are too far away when generating a response. This way, the service provider can learn the area in which the person is, but not where exactly. Cloaking has been used extensively for providing location privacy $2,5,7,9,16$.

\subsubsection{Output Attacks}

There are two kinds of output from the Trusted Module that could reveal location information: Responses to service queries and log entries sent to the Logger Module. For the first kind, we require that the Trusted Module encrypts responses with the operator's public key. For the second kind, we require that log entries do not contain location information. Instead, they contain only information about service queries and about the public key used for encrypting responses to these queries (see Section 5).

Encrypting the response to a service query is not always sufficient to prevent the response from leaking location information. We also require that there always is an output, regardless of a cellphone's location. For example, in a parentchild tracking service, the parent gets alerted when the child leaves a boundary area, where the boundary area is known to the service provider. To implement this service, the Query Processor Module periodically invokes the Trusted Module, which determines whether the current location is outside of the boundary area. If the module returned a response only in the positive case, information would leak to the Query Processor Module. Therefore, the Trusted Module always has to generate a response and the content of the response must not leak any information. For example, the response could be the semantically secure encryption of the value zero or one.

Furthermore, we require that the size of the output of the Trusted Module is determined by the user's cloaked location, not her precise location. For example, when the Trusted Module uses cloaked location information to access the Places Database and filters the records received from the database, the response generated by the module must not allow the provider to learn how many of the records got filtered. See the extended version of this paper 11] for an implementation of this scheme.

Finally, the Trusted Module could output location information using some other means, such as writing the information to a file or to a display. We require that the Trusted Module does not generate output information apart from the output shown in Figure 1 .

\subsubsection{Timing Attacks}

The service provider could measure the delay between giving a service query to the Trusted Module and getting back a response. If different query outcomes resulted in different delays, measuring the delay could leak location information. We require that the processing time of the Trusted Module is independent of the query outcome. For example, for a service that checks for a nearby friend, the delay must be the same, regardless whether the friend is nearby or not. To strictly enforce this property, we would have to guarantee that the number of consumed CPU cycles is identical in either case, which is difficult to enforce in practice. Instead, in our sample location-based services, we ensure that the executed code path is identical in either case and that the only difference is the outcome of one or multiple statements comparing variables. For example, for a nearby-friends service, we have the module compute distance dist between two cellphones and then determine the query response as response $=$ (dist $<=$ nearby)

where nearby indicates the distance considered nearby. In a more complex example, the following statements determine whether a cellphone at position $(\mathrm{x}, \mathrm{y})$ is within a rectangular area $(\mathrm{x} 1, \mathrm{y} 1)-(\mathrm{x} 2, \mathrm{y} 2)$ :

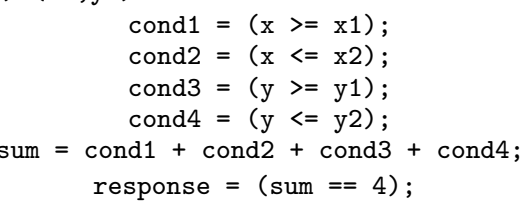

In the extended version of this paper [11, we show that the observed timing differences for the code examples shown above are orders of magnitudes smaller than what is required for a successful timing attack.

\subsubsection{Memory Attacks}

The service provider might try to extract location information or the symmetric decryption key from the memory used by the Trusted Module. We require that the provider cannot access this memory using software-based mechanisms. In our sample location-based services, we deploy the Trusted Module on a dedicated machine and configure the machine such that the provider cannot log in to it.

\subsection{Remote Attestation}

In Section 4.1. we came up with a set of requirements that the Trusted Module must satisfy to prevent location information from leaking. Of course, the developer of the Trusted Module, who could be identical with the service provider, might not actually address these requirements in the implementation. To ensure that our requirements are being satisfied, we use remote attestation.

Remote attestation is a feature proposed by the Trusted Computing Group (TCG) 22. With the help of an inexpensive chip (in TCG terminology, the Trusted Platform Module (TPM)) soldered to the motherboard of a computer, this feature allows an entity to remotely gather information about the code running on this computer. Starting at boot time, each piece of running code (including BIOS code) gathers information about the next piece of code to be loaded and executed and stores this information in the TPM. This process is called a measurement. The TPM guarantees that measurements cannot be reverted (using software-based mechanisms). Therefore, we end up with a chain of measurements, rooted at the static root of trust, which typically is part of the computer's BIOS. If the entity asking a remote computer to perform a remote attestation trusts this root, the TPM, and each piece of loaded code, the entity can retrieve the measurement list from the TPM and infer what code is loaded and executed on the computer. In practice, a TPM does not store a measurement list, only a cryptographic digest, and the actual list is kept by the measuring code (see Sailer et al. 20 for details). A remote attestation returns the list and its digest, signed by the TPM, to the querying entity. A signed digest is called a quote.

Going back to the Trusted Module, the network operator exploits remote attestation to retrieve the measurement list from the module, which will allow the operator to learn the configuration of the module. The operator compares the received list to a list of approved measurements. Software is in an approved state if it is in a given state (e.g., a particular binary with a set of configuration parameters), as in the 
case of a kernel, or if it satisfies the requirements discussed in Section 4.1, as in the case of a component implementing a particular location-based service. To build the list of approved measurements, the operator must have had access to the software (including source code, binaries, and compiler) beforehand for inspection purposes. If the operator can validate the received list of measurements, the operator will hand over the symmetric key that allows decryption of location information to the Trusted Module.

We show the protocol for this transfer in Table 1 it is partially based on earlier work 15, 20. We assume that the configuration of the Trusted Module has been measured in the TPM as part of the boot process. When asking for a remote attestation, the Attestation Module generates a nonce and sends it to the Trusted Module, which generates an asymmetric key pair. The public key, Public Transfer, will be given to the Attestation Module, which will employ it to transfer the symmetric key used for encrypting location information to the Trusted Module. By measuring Public Transfer $_{\text {in the TPM, the Trusted Module guar- }}$ antees to the Attestation Module that the key pair was created by the Trusted Module and that the private key, Private $_{\text {Transfer }}$, is kept within the module and will be erased from memory after the transfer, as a defense against coldboot attacks 8 .

The Trusted Module hands over the nonce received from the Attestation Module to the TPM and requests a quote. The returned quote includes the nonce and is signed with a private key embedded in the TPM (in TCG terminology, the private key of the Attestation Identity Key (AIK) pair). The Attestation Module knows the corresponding public key. The Trusted Module returns the quote, the measurement list, and Public $c_{\text {Transfer }}$ to the Attestation Module, which recomputes the measurement list based on the expected configuration of the Trusted Module. The Attestation Module cannot simply look at the quoted digest, since this value depends on the order in which the OS running on the Trusted Module's computer loads kernel modules and applications, which can differ slightly across reboots. If there are any values in the measurement list that are unknown to the Attestation Module, the module aborts the protocol. Else, the Attestation Module uses Public Transfer to encrypt the symmetric key and sends the ciphertext to the Trusted Module.

An operator might repeat remote attestation periodically (e.g., in an attempt to detect time-of-check, time-of-use (TOCTOU) attacks). Here, the Trusted Module also has to hand over previously used Public $c_{\text {Transfer }}$ so that the Attestation Module can recompute the measurement list.

\section{SECURE LOGGING}

The Trusted Module invokes the Logger Module to keep a $\log$ of its actions. These actions are requested by the Query Processor Module. With the help of the log, we can detect wrongly requested actions, that is, misbehaviour by the Query Processor Module (i.e., the service provider). For example, this module is expected to run access control for some location-based services, such as ensuring that only authorized people can track a person. If the module did not run access control and allowed, for example, the service provider to track the person, a corresponding log entry would make the person aware of this violation, and she would alert the network operator.
An alternative approach is to run access control in the Trusted Module. However, we would like to keep this module as simple as possible, which decreases the likelihood of a vulnerability in the module and also makes it easier for an operator to check the module's correctness. Furthermore, a service provider could be reluctant to implement the entire service within the Trusted Module, since part of this implementation might be proprietary, and the provider does not want to reveal it to an operator for inspection.

Our goal is to log each access to a person's location such that log entries cannot be tampered with or removed. For each access to a person's location, logging should enable this person to learn 1) the service query that triggered this access and 2) the public key that was used for encrypting the response to this query. In Sections 5.1 and 5.2 we discuss how we support these requirements. In Section 5.3, we show how we use the logged information to detect misbehaviour.

\subsection{Logged Information}

A log entry is generated by the Trusted Module whenever it accesses somebody's location. An entry contains an identifier unique for the user whose location was accessed, a cryptographic digest of the service query that triggered this access, and a cryptographic digest of the public key used for encrypting the module's response (see Table 2). It is possible to log additional information, such as the accessed location information (in encrypted form) or the response to a query, but this is not required by our threat model.

The user identifier will be used during log checking to learn whose location information was accessed. This identifier is communicated to the Trusted Module by the Locator Module, together with the (encrypted) location information. A message authentication code protects their integrity.

The query digest is computed by the Query Processor Module and given to the Trusted Module. The Query Processor Module needs to store the corresponding service query.

The Query Processor Module also hands over the operator's public key to the Trusted Module so that the module can encrypt the response to a query. A malicious Query Processor Module could hand over its own public key, which would allow it to decrypt the response. Therefore, the Trusted Module, not the Query Processor Module, computes the digest of the received public key to be included in the log entry. We let the Query Processor Module compute the digest of a (signed) query because the module gains no advantage from cheating here.

A single query can result in multiple log entries. For example, in a nearby-friends service, if Alice tries to locate Bob, the Trusted Module logs accesses to both Alice and Bob's location, each entry containing a digest of Alice's query. The operator inserts a nonce into a query to detect replay attacks by the Query Processor Module (e.g., in a timing attack).

\subsection{Tampering and Removal Attacks}

To protect log entries against tampering, the Trusted Module signs each entry before handing it over to the Logger Module. The Trusted Module logs the public key for the signing key in certified form whenever the module is initialized. The certificate is signed with the TPM's AIK private key. We cannot use this key directly for signing a log entry, since it can be used only for signing keys.

To protect log entries against their removal, the Trusted Module includes the current value of a monotonically in- 
Attestation Module $\rightarrow$ Trusted Module: Trusted Module:

Trusted Module $\rightarrow$ Attestation Module: Attestation Module:

Attestation Module $\rightarrow$ Trusted Module:

Trusted Module: nonce

generate key pair (Public Transfer $_{\text {Trivate }}$ Transfer $)$, measure Public $_{\text {Transfer }}$ in TPM, hand over nonce to TPM, retrieve quote

measurement list $M L, S_{\text {Private }_{A I K}}\left(\right.$ nonce $\left._{\text {|digest }}(M L)\right)$, Public $_{\text {Transfer }}$ check quote signature, re-compute and approve $M L$, abort if failure

$E_{\text {Public }}$ Transfer $($ SymmetricKey)

retrieve SymmetricKey, erase PrivateTransfer

Table 1: Transfer protocol for symmetric key. $S_{F o o}()$ is a signing operation with key Foo, $E_{B a r}()$ an encryption operation with key Bar.

\begin{tabular}{|c|c|c|c|c|c|}
\hline \multicolumn{2}{|c|}{ Counter } & \multirow{2}{*}{\multicolumn{3}{|c|}{ Log entry }} & \\
\hline TPM & Software & & & & \\
\hline 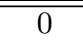 & $\overline{0}$ & \multicolumn{3}{|c|}{ AIK-certified signature verification key } & signature \\
\hline 0 & 1 & user id & query digest & public key digest & signature \\
\hline 0 & 2 & user id & query digest & public key digest & signature \\
\hline 0 & 3 & \multicolumn{3}{|c|}{ special log entry } & signature \\
\hline 1 & 0 & \multicolumn{3}{|c|}{ AIK-certified signature verification key } & signature \\
\hline 1 & 1 & user id & query digest & public key digest & signature \\
\hline 1 & 2 & \multicolumn{3}{|c|}{ special log entry } & signature \\
\hline 2 & 0 & \multicolumn{3}{|c|}{ AIK-certified signature verification key } & signature \\
\hline 2 & 1 & user id & query digest & public key digest & signature \\
\hline
\end{tabular}

Table 2: Log format. A signature covers the counter and the log entry.

creasing counter in each entry and increases this counter after generating an entry. Here, the module needs to be able to reliably determine the value of this counter in case of a restart of the module. A possible approach is to use a TPM v1.2 monotonic counter, which is a counter within the TPM that can never be decreased. A problem with such a counter is that a TPM might support increases only once every five seconds to avoid burnouts. To increase logging frequency, we divide time into epochs. An epoch corresponds to the time between the initialization of the Trusted Module and its shut down. We use a TPM v1.2 monotonic counter to denote the current epoch and a software-based monotonic counter to denote time within an epoch. The Trusted Module includes the current values of both counters in a log entry. The module increases the TPM v1.2 monotonic counter only during module initialization. At the same time, the software-based counter is set to zero. This counter is increased after generating a log entry, which results in a global order among the $\log$ entries.

The above algorithm ensures that, given a log entry, we can detect removal of earlier log entries that have the same TPM v1.2 monotonic counter value. However, the algorithm does not protect against the removal of more recent log entries (i.e., with larger software-based or TPM counter values) or against the removal of log entries with lower TPM v1.2 monotonic counter values. For example, given a log entry with a TPM v1.2 monotonic counter value of 5 , all we can say is there are must be log entries with TPM v1.2 monotonic counter values of $0,1, \ldots, 4$, but not how many of each kind. There could also be log entries with TPM v1.2 monotonic counter values of $6,7, \ldots$ Let us discuss how we address these two attacks.

For the first attack, we want to ensure to a cellphone user that the last log entry seen by her when checking the log is the most recent log entry generated by the Trusted Module for an access to her location information (or close to the most recent one, since new entries can be added while the user is checking log entries). We can satisfy this condition by making the user send a query that accesses her location information to the location-based service before the user checks her log entries. This way, the Trusted Module will create a new log entry, and the user can verify whether there is an entry for her query in the log. If so, the log is up to date.

The second attack can be avoided by having the Trusted Module generate a special log entry whenever the service provider makes the module shut down. This way, an increase in the value of the TPM v1.2 monotonic counter, as observed in the log, is always preceded by such a special entry, and missing log entries can be detected (see Table 2 ). The absence of a special entry does not necessarily imply malicious behaviour. For instance, the Trusted Module could have crashed or there might have been a power outage, which prevented the module from creating the special entry. However, an operator should get suspicious if there are many missing special log entries. Then, either the location-based service is unreliable or there is widespread removal of log entries. Neither of the options is in the operator's interest, and the operator should stop using the service.

\subsection{Log Validation}

Consistent with the architecture described in Section 3. we assume that the operator will take care of the cryptographic operations required for log validation. If a log entry is considered valid, the operator will forward the query that triggered access to a particular user's location to this user for inspection. In more detail, log validation consists of the following steps:

1. The user issues a service query, where processing of the query requires access to her location information. This will create a log entry. 
2. The operator downloads the log (i.e., the entries added since the previous validation) from the service platform. The operator checks the digital signature of each entry and ensures that the counter values are monotonically increasing and complete.

3. The operator determines the log entries relevant to the user based on the user identifier and asks the service provider for the corresponding service queries. The operator ensures that the digests of the received queries match the logged digests and that the queries are properly formed and fresh. The operator also checks whether the query issued in step 1) is in the log.

4. The operator ensures that the logged digests of the public key match the digest of the public key of the operator. If so, the query is forwarded to the user.

5. The user inspects the received queries. If she detects an unauthorized query, she will alert the operator.

\section{SAMPLE LOCATION-BASED SERVICE}

Let us illustrate our architecture by demonstrating how it can be used to implement a service that allows a user to learn whether a friend of hers is nearby. Here, the user sends a query listing the name of the friend to the Forwarder Module, which signs the query, adds the (encrypted) location of the user's cellphone, and forwards it to the Query Processor Module. (Including the location with a query is an optimization to the architecture discussed in Section 3.2.) The Query Processor Module retrieves the friend's policy from the User Database and ensures that the friend has granted access to the user. If so, the module requests the location of the friend's cellphone from the Locator Module. Next, it hands the two locations and the operator's public key over to the Trusted Module.

The Trusted Module uses the symmetric key received during attestation to decrypt the two locations and determines whether they are nearby. Depending on the result, it signs a byte of value either zero or one with its private key, encrypts everything with the operator's public key, and returns the ciphertext to the Query Processor Module, which sends it to the Forwarder Module. This module decrypts the ciphertext and sends the result to the cellphone. The Trusted Module also logs two accesses to the user's and her friend's location.

\section{RELATED WORK}

Research in location privacy has studied how to address the apparent contradiction, where, on the one hand, a locationbased service needs access to location information to be useful, but, on the other hand, it should not have this access because of privacy concerns. A popular approach is location cloaking $2,5,7,9,16$, where only coarse-grained, less intrusive location information is given to a location-based service. Other work 1, 18 has suggested the usage of pseudonyms to keep a person's identity hidden from a service. However, some location-based services do require fine-grained location information or information about the person's identity, else service quality could suffer 2]. Services that alert parents when their child leaves a boundary area or that alert people of nearby friends fall into this category. Our architecture supports such services.
Kölsch et al. 13 also examine location privacy in a setup where the network operator and the service provider are different entities. They introduce a trusted third party that tracks users and that alerts the service provider when the user is in a particular area. The authors do not present an implementation of their concept, in particular, they do not discuss who acts as this third party. Our Trusted Module, as introduced in Section 3 can be looked at as such a third party. Apart from having a proof-of-concept implementation, our architecture has the additional advantage that it can exploit identity information and fine-grained location information, which increases service quality, whereas the previous work relies on coarse-grained location information and pseudonyms. Ravi et al. 19 have a service provider migrate the code that implements a location-based service to a network operator. The operator uses information flow control to ensure that the code does not leak a cellphone's finegrained location to the provider. This approach is targeted at services that exploit aggregate location information and does not support services that require fine-grained location, as supported by our solution.

The usage of Trusted Computing or similar technologies for privacy protection has been proposed in earlier work. Molnar et al. 17. give a secret required for reading RFID tags to a reader only if the reader passes a remote configuration check. There is no implementation of this idea and no discussion of how the operator of a reader might indirectly gain access to data read by the reader. Iliev and Smith [12] deploy a tamperproof coprocessor at a server and use it for implementing a Private Information Retrieval (PIR) scheme 3], which allows database accesses without the owner of the database learning which records are being accessed. We could use PIR to let a cellphone retrieve information about interesting places close to its location without the service provider operating the database learning the location. However, Iliev and Smith's scheme calls for periodic re-shuffles of all the records in the database, which is expensive. Efficient PIR schemes that do not require a tamperproof coprocessor (e.g., by Goldberg 6]) touch all the records in the database for each access. Our compromise, as explained in Section 4.1 is more efficient, but reveals coarse-grained location information to the service provider.

Previous research has explored the usage of Trusted Computing technologies to validate the configuration of a remote server 14, 20. Here, a client ensures that the server (e.g., a web server) has not been tampered with and that it corresponds to an approved version. Our problem is harder because of the presence of the service provider, who can passively and actively intercept queries/responses flowing into/out of the service platform, which might leak location information (see Section 4.1). Moreover, to limit the danger of an exploitable vulnerability being present in the configuration trusted by the client, we do not include an entire server in this configuration, only the part that directly deals with location information. Finally, we give a set of requirements that need to be satisfied in order for a particular version of software getting approval, which is a topic that has been neglected in previous work.

In terms of Secure Logging, Schneier and Kelsey 21 look at logging in the presence of an attacker. However, their threat model is different from ours. They are concerned about an attacker breaking into a machine, like a service provider managing to subvert our architecture, and modify- 
ing existing $\log$ entries. This is not a threat that we study in this paper, so the previous research is orthogonal to ours. A problem that we, but not the previous work, study is how to securely initialize the logging process upon a reboot of the service platform.

\section{CONCLUSIONS AND FUTURE WORK}

We have demonstrated that it is possible to build locationbased services for which the service provider does not become aware of users' fine-grained location. In addition to keeping location information away from a service provider by encrypting it, we have also addressed several other passive and active attacks that a service provider might perform to gain access to this information.

Future work involves studying solutions based on a dynamic root of trust and applying our approach to protect the privacy of personal information other than location information, not necessarily only in location-based services.

\section{Acknowledgments}

We thank the anonymous reviewers for their comments. This work is supported by the Natural Sciences and Engineering Research Council of Canada.

\section{REFERENCES}

[1] A. R. Beresford and F. Stajano. Location Privacy in Pervasive Computing. IEEE Pervasive Computing, 2(1):46-55, 2003.

[2] R. Cheng, Y. Zhang, E. Bertino, and S. Prabhakar. Preserving User Location Privacy in Mobile Data Management Infrastructures. In Proceedings of PET 2006, June 2006.

[3] B. Chor, E. Kushilevitz, O. Goldreich, and M. Sudan. Private Information Retrieval. Journal of the ACM, 45(6):965-981, 1998.

[4] Cybit Ltd. mapAmobile. http://www.mapamobile.com Accessed June 2008.

[5] G. Ghinita, P. Kalnis, and S. Skiadopoulos. PRIVÉ: Anonymous Location-Based Queries in Distributed Mobile Systems. In Proceedings of 16th International World Wide Web Conference (WWW2007), pages 371-380, May 2007.

[6] I. Goldberg. Improving the Robustness of Private Information Retrieval. In Proceedings of IEEE Security and Privacy Symposium, pages 131-148, May 2007.

[7] M. Gruteser and D. Grunwald. Anonymous Usage of Location-Based Services Through Spatial and Temporal Cloaking. In Proceedings of 1st International Conference on Mobile Systems, Applications, and Services (MobiSys 2003), pages 31-42, May 2003.

[8] J. A. Halderman, S. D. Schoen, N. Heninger, W. Clarkson, W. Paul, J. A. Calandrino, A. J. Feldman, J. Appelbaum, and E. W. Felten. Lest We Remember: Cold Boot Attacks on Encryption Keys. In Proceedings of 17th USENIX Security Symposium, July/August 2008

[9] T. Hashem and L. Kulik. Safeguarding Location Privacy in Wireless Ad-Hoc Networks. In Proceedings of 9th International Conference on Ubiquitous Computing (UbiComp 2007), pages 372-390, September 2007.
[10] U. Hengartner. Hiding Location Information from Location-Based Services. In Proceedings of International Workshop on Privacy-Aware Location-based Mobile Services (PALMS), May 2007.

[11] U. Hengartner. Design and Evaluation of an Architecture for Location Privacy. Technical Report CACR 2008-14, Centre for Applied Cryptographic Research, University of Waterloo, July 2008.

[12] A. Iliev and S. W. Smith. Protecting Client Privacy with Trusted Computing at the Server. IEEE Security and Privacy, 3(2):20-28, March/April 2005.

[13] T. Kölsch, L. Fritsch, M. Kohlweiss, and D. Kesdogan. Privacy for Profitable Location Based Services. In Proceedings of 2nd International Conference on Security in Pervasive Computing (SPC 2005), pages 164-178, April 2005.

[14] J. Marchesini, S. W. Smith, O. Wild, J. Stabiner, and A. Barsamian. Open-Source Applications of TCPA Hardware. In Proceedings of 20th Annual Computer Security Applications Conference (ACSAC'04), pages 294-303, December 2004.

[15] J. M. McCune, B. Parno, A. Perrig, M. K. Reiter, and A. Seshadri. Minimal TCB Code Execution. In Proceedings of IEEE Security and Privacy Symposium, pages 267-272, May 2007.

[16] M. F. Mokbel, C.-Y. Chow, and W. G. Aref. The New Casper: Query Processing for Location Services without Compromising Privacy. In Proceedings of 32nd International Conference on Very Large Data Bases (VLDB 2006), pages 763-774, September 2006.

[17] D. Molnar, A. Soppera, and D. Wagner. RFID Privacy Through Trusted Computing. In Proceedings of Workshop on Privacy in the Electronic Society (WPES'05), November 2005.

[18] G. Myles, A. Friday, and N. Davies. Preserving Privacy in Environments with Location-Based Applications. Pervasive Computing, 2(1):56-64, January-March 2003.

[19] N. Ravi, M. Gruteser, and L. Iftode. Non-Inference: An Information Flow Control Model for Location-based Services. In Proceedings of $3 r d$ International Conference on Mobile and Ubiquitous Systems: Networks and Services (MobiQuitous 2006), July 2006.

[20] R. Sailer, X. Zhang, T. Jaeger, and L. van Doorn. Design and Implementation of a TCG-based Integrity Measurement Architecture. In Proceedings of 13th USENIX Security Symposium, August 2004.

[21] B. Schneier and J. Kelsey. Cryptographic Support for Secure Logs on Untrusted Machines. In Proceedings of 7th USENIX Security Symposium, pages 53-62, January 1998.

[22] Trusted Computing Group. https://www.trustedcomputinggroup.org. Accessed June 2008.

[23] WaveMarket, Inc. Family Finder. http://www. wavemarket.com. Accessed June 2008.

[24] World-Tracker.Com. http://www .world-tracker.com Accessed June 2008 